\title{
Temporal kinetics of prefrontal modulation of the extrastriate cortex during visual attention
}

\author{
ELENA YAGO, AUDREY DUARTE, and TING WONG \\ University of California, Berkeley, California \\ FRANCISCO BARCELÓ \\ University of the Balearic Islands, Palma de Mallorca, Spain \\ and \\ ROBERT T. KNIGHT \\ University of California, Berkeley, California
}

\begin{abstract}
Single-unit, event-related potential (ERP), and neuroimaging studies have implicated the prefrontal cortex (PFC) in top-down control of attention and working memory. We conducted an experiment in patients with unilateral PFC damage $(n=8)$ to assess the temporal kinetics of PFC-extrastriate interactions during visual attention. Subjects alternated attention between the left and the right hemifields in successive runs while they detected target stimuli embedded in streams of repetitive task-irrelevant stimuli (standards). The design enabled us to examine tonic (spatial selection) and phasic (feature selection) PFC-extrastriate interactions. PFC damage impaired performance in the visual field contralateral to lesions, as manifested by both larger reaction times and error rates. Assessment of the extrastriate P1 ERP revealed that the PFC exerts a tonic (spatial selection) excitatory input to the ipsilateral extrastriate cortex as early as 100 msec post stimulus delivery. The PFC exerts a second phasic (feature selection) excitatory extrastriate modulation from 180 to $300 \mathrm{msec}$, as evidenced by reductions in selection negativity after damage. Finally, reductions of the N2 ERP to target stimuli supports the notion that the PFC exerts a third phasic (target selection) signal necessary for successful template matching during postselection analysis of target features. The results provide electrophysiological evidence of three distinct tonic and phasic PFC inputs to the extrastriate cortex in the initial few hundred milliseconds of stimulus processing. Damage to this network appears to underlie the pervasive deficits in attention observed in patients with prefrontal lesions.
\end{abstract}

The limited capacity of the brain for the processing of sensory inputs requires mechanisms that select relevant information from the complex sensory environment. The selection of a specific aspect of the visual scene, such as location, color, or shape, leads to enhanced activation of extrastriate visual areas. It has been proposed that selective attention acts as an amplifier that enhances the neural activity in the cortical zones that encode and represent the attended feature (Corbetta, Miezin, Dobmeyer, Shulman, \& Petersen, 1991; Posner \& Dehaene, 1994). Electrophysiological and neuroimaging studies in humans and monkeys have shown that attentional modulation is observed in extrastriate areas in the occipital-temporalparietal cortices, as reflected by attention-related modula-

This study was supported by NIH Grants NS21135 and PO NS40813 and by Grant PR2004-0012 from the Spanish Ministry of Education and Science. We thank Clay Clayworth for his technical support and the patients and controls for their participation. All of the authors are affiliated with the Helen Wills Neuroscience Institute at the University of California, Berkeley. Correspondence concerning this article should be addressed to R. T. Knight, Helen Wills Neuroscience Institute, University of California, 130 Barker Hall, Berkeley, CA 94729-3190 (e-mail: rtknight@socrates.berkeley.edu). tions of event-related potentials (ERPs), as well as positron emission tomography (PET), and functional magnetic resonance imaging (fMRI) signals (Brefczynski \& DeYoe, 1999; Corbetta et al., 1991; Haxby et al., 1994; Heinze et al., 1994; Reynolds, Pasternak, \& Desimone, 2000; Sereno et al., 1995). When attention has to be directed to one of the visual hemifields, attentional modulation of extrastriate activity is preferentially observed over the hemisphere contralateral to the attended hemifield (Clark \& Hillyard, 1996; Heinze et al., 1994; Mangun, Buonocore, Girelli, \& Jha, 1998; Mangun \& Hillyard, 1993; Vandenberghe et al., 1997; Woldorff et al., 1997).

It has been proposed that the attentional modulation of visual extrastriate areas is controlled by higher order brain structures, such as the prefrontal and parietal association cortices (Corbetta, 1998; Hillyard \& Anllo-Vento, 1998). Association cortex dependent top-down modulation of visual areas is clearly observed when activity in the visual cortex increases in the absence of visual stimulation, such as by the presence of visual cues after a delay period, indicating the subsequent appearance of a visual target (Kastner, Pinsk, de Weerd, Desimone, \& Ungerleider, 1999). Indeed, studies based on single-cell recordings (Rainer, 
Asaad, \& Miller, 1998; Tomita, Ohbayashi, Nakahara, Hasegawa, \& Miyashita, 1999), lesion studies (Barceló, Suwazono, \& Knight, 2000; Knight, 1997; NielsenBohlman \& Knight, 1999), and functional brain imaging (Büchel \& Friston, 1997; Chawla, Rees, \& Friston, 1999; Corbetta, Miezin, Shulman, \& Petersen, 1993; de Fockert, Rees, Frith, \& Lavie, 2001; Kastner et al., 1999; McIntosh et al., 1994; Rees, Frackowiak, \& Frith, 1997; Tootell et al., 1998) provide evidence that the prefrontal cortex (PFC) modulates extrastriate processing. According to Hillyard and Anllo-Vento (1998), this attentional control network provides bias signals that either enhance or suppress sensory representations in the extrastriate visual pathways according to their momentary behavioral relevance.

Electrophysiological techniques can be used to track the temporal course of attentional modulation of visual information flow (for reviews, see Hillyard, Mangun, Woldorff, \& Luck, 1995; Mangun, 1995). This modulation begins at early stages of visual processing, as reflected in an enhanced amplitude to attended, as compared with unattended, visual locations for the sensory-evoked potentials P1 (80-120 msec post stimulus onset) and N1 (140-190 msec; Hillyard \& Anllo-Vento, 1998; Hillyard et al., 1995; Luck, 1995; Mangun et al., 1998; Wijers, Lange, Mulder, \& Mulder, 1997). P1 generation has been localized over the ventral-lateral occipital scalp, by means of current source density analyses and in the extrastriate cortex through dipole modeling (Clark \& Hillyard, 1996; Mangun \& Hillyard, 1993). The N1 has been localized over the occipital-parietal cortex (Mangun et al., 1998). The P1 and N1 potentials reflect the early stages of visual processing preceding full perceptual analysis (Hillyard \& Anllo-Vento, 1998; Mangun, 1995; Wijers et al., 1997).

The modulation of these early ERP components has been related to spatial attention, rather than to the analysis of object features, such as color (Hillyard \& Münte, 1984; Wijers, Mulder, Okita, Mulder, \& Scheffers, 1989), orientation (Smid, Jakob, \& Heinze, 1999), or spatial frequency (Hillyard et al., 1995). These early ERP effects of spatial attention have been accounted for in terms of a sensory gain mechanism, because spatial attention enhances the early exogenous components (e.g., P1 and N1), without changing their latency or scalp topography (Baas, Kenemans, \& Mangun, 2002; see Mangun, 1995, for a review). These spatial ERP attention effects are often more robust for the N1 component.

In contrast, the ERP signature of selective processing of nonspatial object features is a negative polarity occipital ERP component starting at about $150 \mathrm{msec}$ poststimulus-onset, and overlapping partially with the early exogenous components. This component was initially labeled selection negativity (SN) by Harter and colleagues (Harter \& Aine, 1984). The SN is a distinct component related to the analysis of specific features of attended stimuli (Baas et al., 2002; Smid et al., 1999). For instance, if one is asked to attend only to a specific color stimulus in a train of stimuli, only the attended color stimulus of interest elicits an SN. In a spatial attention task such as that employed in the present experiment, all the stimuli in the attended field would be expected to elicit an SN (Baas et al., 2002; Smid et al., 1999).

It has been proposed that this early modulation of visual processing allows inputs from attended regions of the visual field to gain preferential access to higher states of feature analysis, pattern identification, and object recognition (Heinze et al., 1994). Postperceptual selection of a specific object in an attended field requires further processing stages indexed by longer latency ERP components, such as the target-related N2 $(250-350 \mathrm{msec})$, which is associated with discrimination and template matching of relevant stimulus dimensions, and the target $\mathrm{P} 3 \mathrm{~b}$ component (350-600 msec), which is triggered when task-relevant events are recognized and correctly classified (Hillyard et al., 1995).

Although top-down modulation of extrastriate areas by the PFC during visual attention is supported by numerous neuroimaging studies, there is limited evidence concerning the temporal parameters of this modulation. Barceló et al. (2000) addressed this issue by recording electrophysiological activity over the extrastriate cortex in a group of patients with lesions involving the lateral PFC while the patients performed a bifield target detection task. In Barceló et al.'s study, the subjects were required to attend and detect targets in both visual fields. The early latency P1 response elicited by standard and target stimuli was reduced in the prefrontal patients only over ipsilesional parietal and temporo-occipital areas. In addition, the patients showed attenuated N2 and P3b target-related responses, which suggested both early and longer latency impaired attentional modulation in these patients. Behavioral performance as measured by reaction times (RTs) and accuracy was impaired in the visual field contralateral to PFC damage. These results provided evidence of an ipsilesional deficit in prefrontal modulation of the extrastriate cortex that resulted in failures in behavioral detection capacity for the contralateral visual field. However, since attention was deployed to all the stimuli in the visual field, the authors could not disentangle whether the patients' ERP reductions corresponded to a phasic (object selection) or a tonic (spatial selection) deficit (Chawla et al., 1999; Kastner et al., 1999; Mangun, 1995; Martinez et al., 1999; Rees et al., 1997; Valdes-Sosa, Bobes, Rodríguez, \& Pinilla, 1998).

In the present study, we recorded ERP activity over the extrastriate cortex in a group of patients with unilateral damage in the lateral PFC while the patients performed a task similar to that used by Barceló et al. (2000). In contrast to Barceló et al., in the present study, the subjects were required to allocate attention to only one visual field at a time. This design permitted us to investigate whether the early $(<200 \mathrm{msec})$ modulation of the extrastriate cortex reflects a tonic (spatial selection) or a phasic (object selection) input, possibly originating in PFC structures. We predicted that if the PFC exerts a tonic, location-independent modulation on extrastriate regions, PFC patients would show decreased extrastriate responses to stimuli appearing in both the attended and unattended visual fields. In contrast, if PFC exerts a phasic, location-dependent mod- 
ulation on the visual cortex, the patients would show reduced extrastriate responses to events presented only in the attended, but not in the unattended, visual hemifield.

\section{METHOD}

\begin{abstract}
Subjects
Eight patients (mean age, $69.8 \pm 10.8$ years; range, $53-82$ years; 3 women and 5 men) were selected on the basis of unilateral focal lesions in the lateral PFC ( 2 right and 6 left), as determined by computed tomography or MRI scanning. Lesions were due to single infarction of the middle cerebral artery ( 7 patients) or craniotomy (1 patient) and were centered in the lateral PFC. Maximal lesion overlap comprised Brodmann's areas $6,8,9,10$, and 46, with variable amounts of damage in Brodmann's areas 44, 45, and 47 (Figure 1). All the patients were at least 1 year postneurological damage. The patients were free of medical complications, psychiatric disorders, substance abuse, psychoactive drug treatment, or other neurological diseases. The patients were compared with 11 age-matched healthy controls (mean age, $69 \pm 7.4$ years; range, $59-82$ years; 3 women and 8 men) who were selected on the basis of the absence of neurological or psychiatric diseases. All the subjects in both groups were right-handed, although 3 patients with lesions over the left hemisphere had upper motor neuron weakness in the right (contralesional) limbs and performed the task with their nondominant (ipsilesional) hands. For this reason, 5 control subjects were asked to respond with their nondominant hands and 6 with their dominant hands. All the subjects gave their informed written consent before participating in the study. This research was approved by the Human Subjects Review Committees of the Martinez Veterans Administration and the University of California, Berkeley.
\end{abstract}

\section{Stimuli and Procedure}

The paradigm used in the present study was adapted from Suwazono, Machado, and Knight (2000). The subjects sat in a comfortable chair located $1.6 \mathrm{~m}$ from a video monitor in a sound-attenuated and dimly lit booth. They were instructed to fixate their gaze on a centrally presented cross and to concentrate their attention in one of the two hemifields of vision (i.e., the left or the right side of the screen, in separate blocks) and to ignore the other hemifield. The task consisted of pressing a response button when a target stimulus appeared on the attended side of the screen. Target stimuli were downside white triangles that appeared with a low probability $(p=.2)$ embedded in a stream of frequent standard stimuli (upside white triangles; $p=.7$ ) and infrequent novel stimuli (unique neutral colorful pictures selected from the International Affective Picture system; $p=.1$ ), as can be seen in Figure 2. The duration of the stimuli was $107 \mathrm{msec}$, and the interstimulus interval was pseudorandomized at 200,800 , or $1,000 \mathrm{msec}$. The stimuli appeared $5^{\circ}$ to either the left or the right side of a central fixation point on a black screen. Standards, targets, and novel stimuli were randomized, as was noted above, with the exception that two targets were never presented sequentially. Thus, the stimuli appeared randomly on either side of the central fixation point.

The subjects were encouraged to respond as quickly and as accurately as possible, to avoid movements and blinks, and to maintain their gaze on the central fixation cross. A practice block was performed before the experiment, to ensure that the subjects clearly understood the task. If necessary, an additional practice block was administered. The recordings consisted of 10 blocks that lasted about 5 min, each containing 270 stimuli. There was a break implemented in the middle of each block and between blocks in order to avoid fatigue. The order of the blocks was counterbalanced across subjects.

\section{Electrophysiological Recording}

Brain electrical activity was recorded from the scalp by 20 tin electrodes, according to the 10-20 system (Fp1, Fp2, F7, F3, Fz,

\section{FRONTAL LESIONS}

R.C.

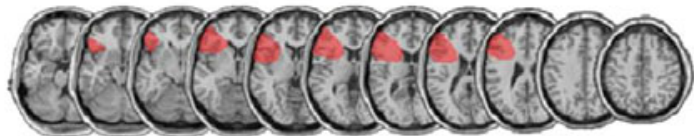

W.E.

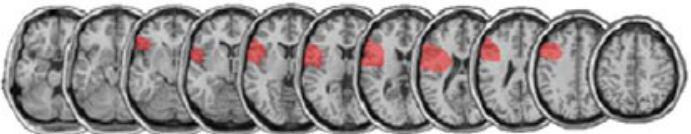

M.F.

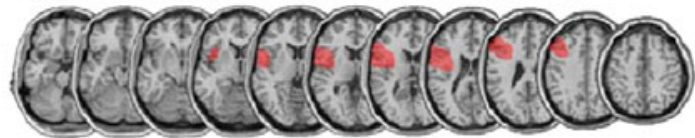

J.C.

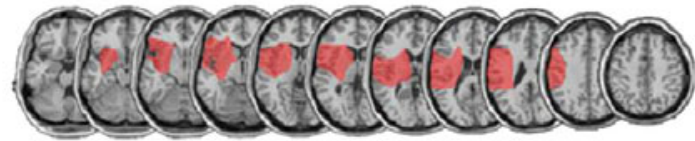

J.M.

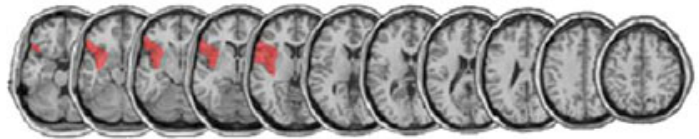

W.A.

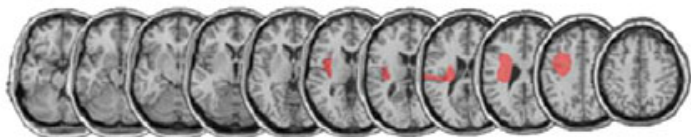

S.R.

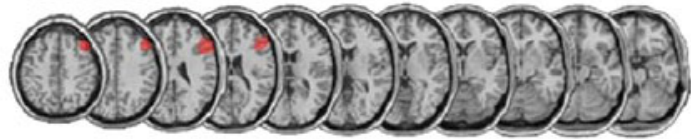

E.B.
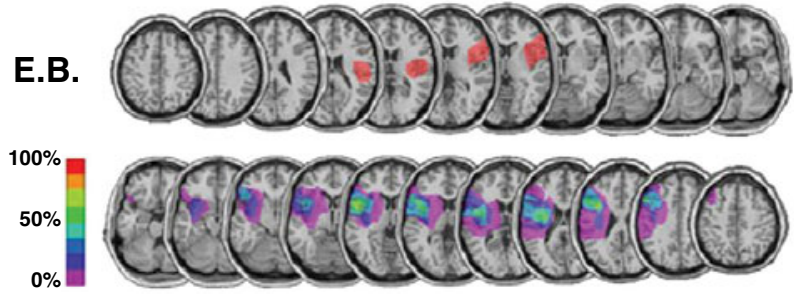

Figure 1. Magnetic resonance imaging (MRI) reconstructions of the lesioned brain areas of each of the 8 patients who participated in the study (patients' initials are given at left). Maximal lesion overlap comprised Brodmann's areas $6,8,9,10$, and 46, with variable amounts of damage in Brodmann's areas 44, 45, and 47. The averaged lesioned area is shown in the bottom row. MRI reconstructions were obtained with MRIcro, compliments of Chris Rorden (www.psychology.nottingham.ac.uk/staff/cr1/mricro. html).

F4, F8, T7, C3, Cz, C4, T8, P7, P3, Pz, P4, P8, O1, Oz, and O2). Ten additional positions were included ( $\mathrm{Ft} 7, \mathrm{Fc} 3, \mathrm{Fcz}, \mathrm{Fc} 4, \mathrm{Ft} 8$, Tp7, Cp3, Cpz, Cp4, and Tp8), as well as the right (RM) and left (LM) mastoids. Eye movements were monitored by two electrodes placed below (vertical electrooculogram) and at the outer canthus (horizontal electrooculogram) of the left eye. All electrode sites were referenced to an electrode attached to the tip of the nose. Electrode impedance was kept below $5 \mathrm{k} \Omega$ at all locations.

\section{Data Analysis}

The EEG was amplified (bandpass, $0.1-100 \mathrm{~Hz}$ ), digitized $(500 \mathrm{~Hz}$ per channel), and digitally stored in a PC for off-line analysis. The averaging epoch was $1,000 \mathrm{msec}$, including $200 \mathrm{msec}$ of prestimulus baseline. In order to avoid artifacts, trials exceeding $\pm 100 \mu \mathrm{V}$ were automatically rejected from further analysis. Individual ERPs were 


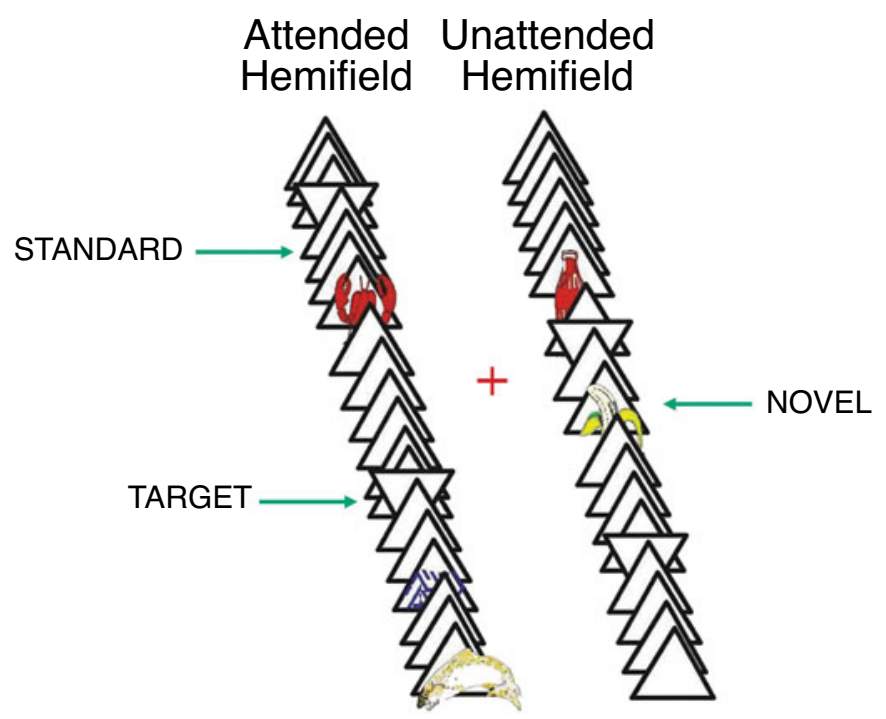

Figure 2. Representation of the stimulation paradigm used in the study.

averaged separately according to the visual hemifield (ipsilesional/ contralesional), experimental condition (attended/unattended), and the type of stimulus (standard/target/novel). Averaged data were bandpass filtered between 0.01 and $15 \mathrm{~Hz}$ and rereferenced to linked mastoids.

Ipsilesional electrophysiological activity was estimated by averaging the ERPs at P7 in left-lesioned patients with those collected from $\mathrm{P} 8$ in right-lesioned patients (parieto-occipital ipsilesional [POi]). Ipsilesional ERP data from the patients were compared with ERP data from the left (P7) of the controls. Only ERPs measured from ipsilesional regions in response to stimuli appearing on the contralesional visual hemifield will be reported here. As in a previous study (Barceló et al., 2000), here the analysis of ipsilesional standards did not reveal any group differences in ERP amplitudes for either the attended or the unattended locations. The P7/P8 electrodes were chosen since they had the largest extrastriate ERP amplitudes and examination of singleelectrode data provides the most stringent statistical criterion. Mean amplitudes from the full array of 30 active electrodes were also obtained to compute the topographical distribution of the ERPs of interest.

Mean amplitudes of ERP components were measured relative to the prestimulus baseline. The mean amplitude of the early ERP activity evoked by standard stimuli was computed in 100-msec time windows around the maximum peaks of $\mathrm{P} 1(60-160 \mathrm{msec})$ and $\mathrm{N} 1$ (120-220 msec) and the longer latency SN (150-350 msec), as identified in the grand averages. Differences between groups and conditions for the mean amplitudes of P1, N1, and SN in POi elicited by contralesional standard stimuli were submitted to a repeated measures analysis of variance (ANOVA) with group (patients vs. controls) as the between-subjects factor and condition (attended vs. unattended) as the within-subjects factor. Greenhouse-Geisser correction for the degrees of freedom was applied when necessary. The uncorrected degrees of freedom and the corrected $p$ values are reported.

Target-related brain activity was studied in the target minus standard ERP difference waves from the contralesional attended hemifield. Mean amplitudes for the $\mathrm{N} 2$ and $\mathrm{P} 3 \mathrm{~b}$ responses in POi were computed between 250 and $350 \mathrm{msec}$ for the N2 and between 400 and $600 \mathrm{msec}$ for the target $\mathrm{P} 3 \mathrm{~b}$. Groups were then compared using a series of $t$ tests for each component. The novelty $\mathrm{P} 3$ response was attenuated in the frontal patients, as has been reported in numerous other studies, and will not be discussed further (Daffner et al., 2003; Knight, 1984, 1997).
RTs, hit rates, and false alarms were computed for each subject in each of the blocks. Buttonpresses between 300 and $700 \mathrm{msec}$ after the appearance of a target in the attended hemifield were considered correct responses. Buttonpresses performed after this time interval were considered missed responses. Responses made after a target was presented in the unattended hemifield, as well as those made in the absence of a target, were considered false alarms. RTs were computed only for correct hits. RTs and hit, miss, and false alarm rates were subjected to an ANOVA for repeated measures, with group (patient vs. control) as the between-subjects factor and hemifield of stimulus presentation (ipsilesional vs. contralesional) and type of stimuli (standard vs. target) as the repeated measures factors.

\section{RESULTS}

\section{Behavior}

The patients were less accurate, having a lower rate of correct responses than the controls did [85.2 vs. 94.4; $F(1,17)=6.24, p<.03$ ], especially evident when targets appeared on the contralesional visual hemifield [interaction of visual hemifield and group: $F(1,17)=5.25$, $p<.04]$. The less accurate performance of the patients was due to a larger number of missing responses to targets presented on the contralesional visual hemifield [interaction of visual hemifield and group: $F(1,17)=$ $5.08, p<.04$; Figure 3, right panel]. No differences were found between the patients and the controls in the number of false alarms $[F(1,17)=1.52, p=.23]$. The patients were slower than the controls when performing the task [501 vs. $447 \mathrm{msec} ; F(1,17)=6.01, p<.03$ ], especially when the targets appeared on the contralesional visual hemifield [interaction of visual hemifield and group: $F(1,17)=4.12, p<.06$; Figure 3, left panel]. These results reveal an attentional impairment in the frontallesioned patients, which is more evident when attention has to be directed to the contralesional visual hemifield. 


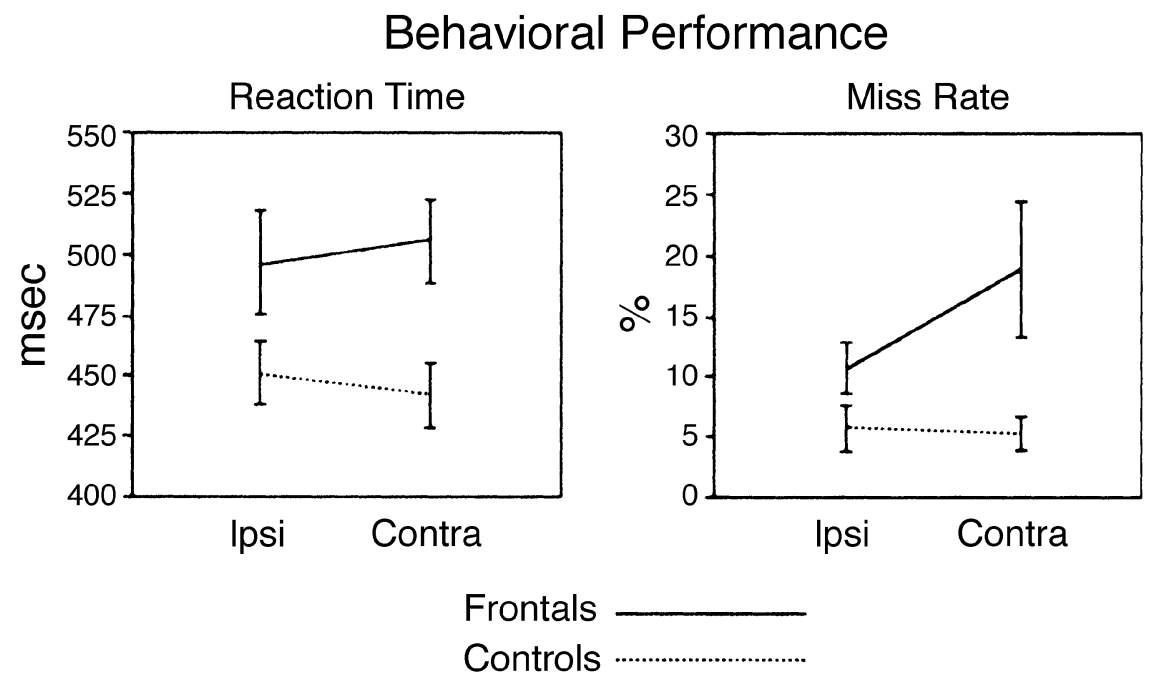

Figure 3. Reaction times and percentages of missing responses in the patients (thick line) and the controls (dashed line) to target stimuli presented in the ipsilesional and contralesional visual hemifields. Vertical bars show standard errors of the means.

\section{Electrophysiology}

ERPs to standards. Standard stimuli elicited the P1 (ca. $100 \mathrm{msec}$ ), N1 (ca. $170 \mathrm{msec}$ ), and SN (ca. $250 \mathrm{msec}$ ) over parieto-occipital electrodes in both groups (Figure 4). The mean amplitude of P1 to standard stimuli was significantly smaller in the patients, as compared with the controls, for both attended and unattended stimuli [patients, $-0.06 \mu \mathrm{V}$; controls, $0.57 \mu \mathrm{V}$; group effect, $F(1,17)=9.05, p<.01]$. A nonsignificant $\mathrm{P} 1$ deflection was noted in the difference waves for both the controls and the patients [attention effect, $F(1,17)=0.76$, $p=.39$; interaction of group and attention, $F(1,17)=$

\section{Contralesional Standard}

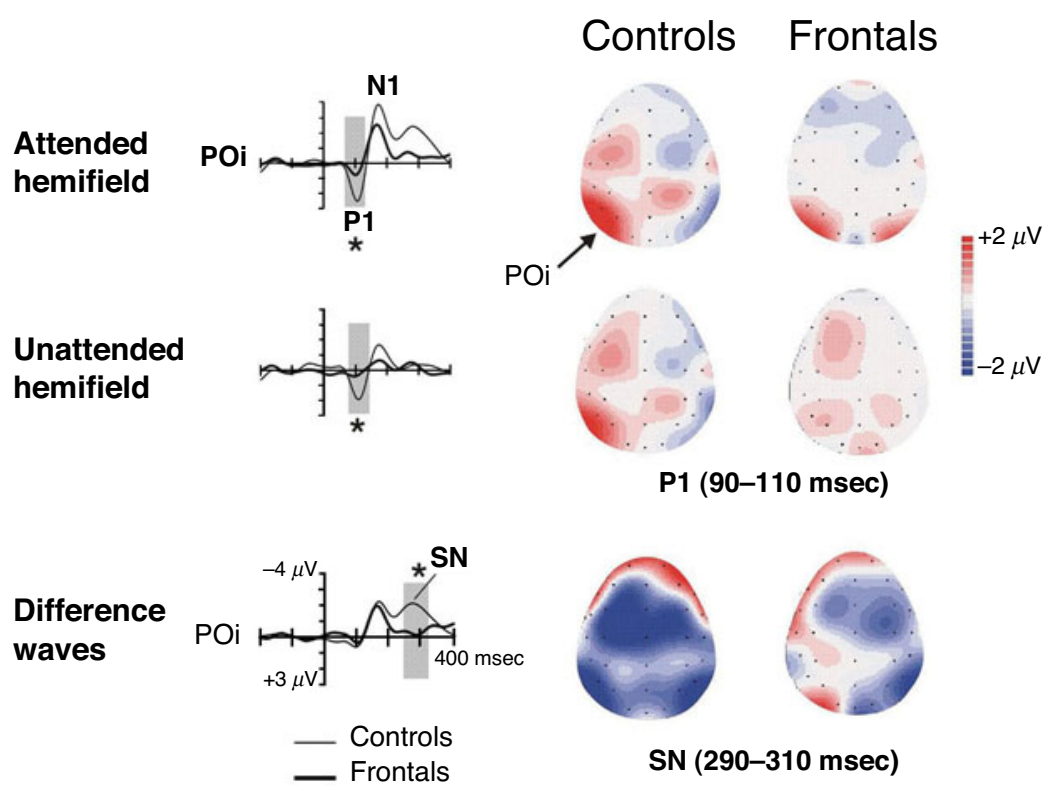

Figure 4. Grand average of the early latency event-related potentials (ERPs) in the group of patients (thick line) and the control group (thin line) to standard contralesional stimuli, at the electrode parieto-occipital ipsilesional (POi). Top: attended hemifield. Middle: unattended hemifield. Bottom: attended minus unattended difference waves. Topographical maps of the distribution of the ERPs of interest (shaded gray window) are shown on the right. 
$1.34, p=.26]$, suggesting that the $\mathrm{P} 1$ was not significantly modulated by attention to location in either group (Figure 4, bottom panel). Following the P1, the mean amplitude of N1 around its maximal peak (120- to 220-msec time window) did not differ between groups [group effect, $F(1,17)=0.61, p=.45]$. Analysis of the difference waves revealed that the $\mathrm{N} 1$ was larger for attended than for unattended stimuli $[F(1,17)=8.14, p<.02]$, in both groups [patients, $-1.46 \mu \mathrm{V}$ vs. $-0.32 \mu \mathrm{V}$; controls, $-1.93 \mu \mathrm{V}$ vs. $-0.69 \mu \mathrm{V}$; interaction of attention and group, $F(1,17)=0.013, p=.91$; Figure 4 , left panel]. In turn, the patients showed reduced activation in the latency range of the $\mathrm{SN}$ response $(150-350 \mathrm{msec})$ to standard stimuli [patients, $-0.25 \mu \mathrm{V}$; controls, $-1.71 \mu \mathrm{V} ; t(17)=$ $2.15 ; p<.05]$, as is reflected in the difference waves from attended minus unattended visual fields (Figure 4, bottom panel). Voltage maps in Figure 4 (bottom panel) show the posterior extrastriate distribution of the SN component, which develops in parallel with two other selection-related ERP features: a far frontal selection positivity (FSP; $120-300 \mathrm{msec}$ ) and a central negativity (N2b; 250-350 msec; cf. Smid et al., 1999), which were both reduced in the patients.

ERPs to targets. The target-related N2 response was also attenuated in the group of patients, as reflected in the target minus standard difference waves [patients, $1.19 \mu \mathrm{V}$; controls, $-2.36 \mu \mathrm{V} ; t(17)=3.5, p<.005$; Figure 5]. No statistical differences between groups were found at later latencies (350-550 msec), corresponding to the target-related $\mathrm{P} 3 \mathrm{~b}$ response [controls, $3.24 \mu \mathrm{V}$ vs. patients, $3.06 \mu \mathrm{V} ; t(17)=-0.17, p=.86$; Figure 5].

\section{DISCUSSION}

In the present study, we investigated the temporal dynamics of top-down modulation of the PFC over the visual extrastriate cortex, using a combined neuropsychological and electrophysiological approach. The behavioral and electrophysiological data revealed three distinct deficits in the modulation of extrastriate activity in the PFC- lesioned patients. First, an early location-independent modulation was related to spatial selection of information at the attended visual hemifield. Second, an early locationdependent modulation was related to the selection of object features. Third, a late location-dependent modulation was related to postselection of target features.

\section{Early Location-Independent Modulation of Extrastriate Activity}

Electrophysiological data revealed decreased activation of visual areas in the group of prefrontal-lesioned patients at early stages of visual processing in the hemisphere ipsilateral to damage, as reflected by the attenuation of the $\mathrm{P} 1$ response $(60-160 \mathrm{msec}$ post stimulus onset). This reduction of activation at the ipsilesional extrastriate cortex was found for stimuli presented in the contralesional visual field, whether or not that field was attended. The results parallel those in Barceló et al. (2000), in which an attenuation of the early evoked response P1 to standard stimuli was also found in a similar sample of patients. However, in the study of Barceló et al., attention was deployed to both visual hemifields, and the authors could not interpret whether the P1 attenuation was a deficit in prestimulus (tonic) or stimulus-locked (phasic) attentional mechanisms. In the present study, P1 was attenuated to both attended and unattended locations, confirming that the attenuation of the sensory-evoked $\mathrm{P} 1$ response was due to a loss of tonic PFC input to the extrastriate cortex. A tonic bilateral PFC modulation of extrastriate regions seems necessary for early spatial selection. This early bilateral PFC influence upon the extrastriate cortex would allow construction of a salience map of all spatial locations (Treue, 2003), even though higher activation occurs at cortical regions contralateral to the attended location. This would explain why the early tonic PFC modulation of the extrastriate cortex was location independent (i.e., prior to selection of a spatial location). This finding supports the notion that the lateral PFC is involved in the maintenance of activation of the extrastriate cortex in the early stages of visual processing.

\section{Contralesional Targets}

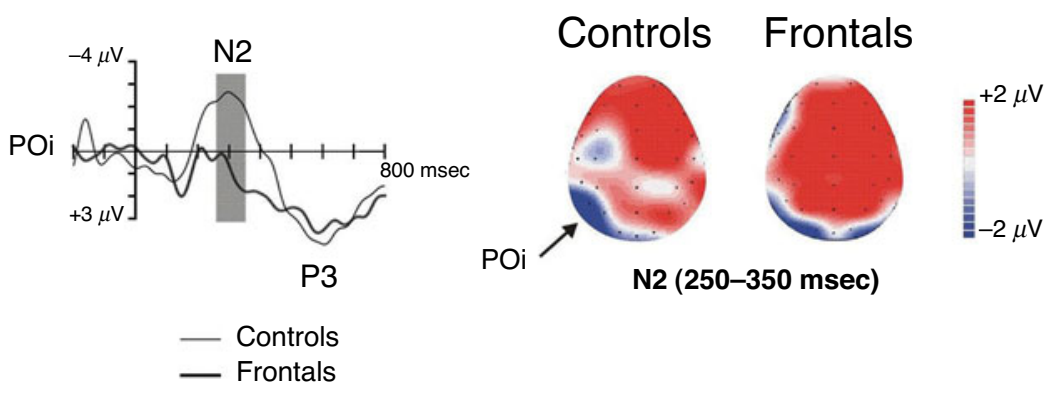

\footnotetext{
Figure 5. Target minus standard difference waves in the attended hemifield, for the group of patients (thick line) and the control group (thin line), at the electrode parietooccipital ipsilesional (POi) to contralesional stimuli. On the right is the scalp distribution of the $\mathrm{N} 2$ (shaded latency window).
} 
Single-cell studies have reported that prefrontal neurons discharge tonically even in the absence of stimulation, probably reflecting active maintenance of an attentiondependent template in working memory (Desimone \& Duncan, 1995; Miller, 1999; Rainer et al., 1998). It has been proposed that the role of the PFC in visual attention involves initiating and maintaining the selective sensory bias in working memory (Hillyard \& Anllo-Vento, 1998). Indeed, in an fMRI study (de Fockert et al., 2001), larger activations over the visual cortex to distracting visual stimuli were found in those conditions in which the working memory load in a concomitant task was larger. The present study supports the concept that the lateral PFC is crucial for the tonic activation of the extrastriate cortex during attention tasks. One might predict that this tonic modulation depends on the PFC-controlled glutamatergic input into the extrastriate cortex from fiber bundles in the inferior or superior longitudinal fasciculus originating in the PFC and terminating in the extrastriate cortices.

\section{Early Location-Dependent Modulation of the Extrastriate Cortex}

Two types of early attentional modulations of the extrastriate cortex were observed in the controls, each with their distinct functional properties. One was indexed by an increase of the P1-N1 responses to attended, as compared with unattended, locations and was preserved in the PFC patients. The other marker of early attention modulation was the SN (150-350 msec), which reflects processing of object features in an unattended channel (i.e., standards, in our task). The SN was markedly reduced over the ipsilesional extrastriate cortex of the prefrontal patients.

Attention modulation of early P1-N1 ERP components has been related to spatial attention, rather than to the analysis of object features, such as color or spatial frequency (Hillyard et al., 1995). The present results suggest that some early aspects of spatial selection were spared after PFC lesions. The behavioral data partially support this assumption, since the selection of the spatial locations was correctly performed by the patients, as revealed by a low rate of false alarms, which would have been triggered by a target stimulus appearing in the unattended visual field. These results of preserved P1-N1 attention enhancements strongly suggest that the PFC is not the only source for the attentional modulation of the visual cortex at early stages of visual processing (up to $180-200 \mathrm{msec}$ ), mainly related to spatial selection of visual information. Consequently, other brain areas must account for this spatial attention modulation. The parietal cortex is the leading candidate for modulating early activity at the extrastriate cortex during spatial attention. Indeed, the posterior parietal cortex is crucial in representing and selecting spatial locations (for a review, see Mesulam, 1999). Furthermore, neuroimaging studies have shown the involvement of the posterior parietal cortex during visual spatial orienting (Corbetta, Kincade, Ollinger, McAvoy, \&
Shulman, 2000; Corbetta et al., 1993; Gitelman et al., 1999; Hopfinger, Buonocore, \& Mangun, 2000).

In sharp contrast, prefrontal lesions completely abolished an SN wave over the ipsilesional extrastriate cortex in the patients. The SN has been proposed to reflect residual processing of object features in an unattended channel (i.e., in task-irrelevant standards), after the early operation of the P1-N1 gating mechanisms (Smid et al., 1999). Hence, the $\mathrm{SN}$ would reflect a postselection analysis of those aspects of stimulation that are task relevant. This postselection analysis is a key process for feature integration and the construction of the visual percept (Friedman-Hill, Robertson, \& Treisman, 1995; Smid et al., 1999). Disruption of this process might partly account for the deficits of attention observed here in the prefrontal patients. As in many real-life situations, PFC patients can probably use their intact hemisphere to partly compensate for their attentional deficits.

Although not the focus of the present report, the SN component developed in parallel with two concurrent selection-related ERP features that appeared to be disrupted in patients: a far FSP and a mid-central negativity (N2b; see Figure 4; cf. Smid et al., 1999). The former concerns a slow endogenous positive shift, maximal over the frontolateral scalp (Smid et al., 1999). The latter concerns a mid-central negativity that onsets after the $\mathrm{SN}$ and FSP and has been named N2b (Smid et al., 1999; Wijers et al., 1989). Further work will be necessary to disclose the exact role played by the PFC in coordinating these three types of selective attention processes. Our present results emphasize the crucial role of the PFC as part of a distributed network of distinct brain regions, each supporting a different functional aspect of the selection of task-relevant information indexed by neural activity in the extrastriate cortex (Baas et al., 2002; Smid et al., 1999).

\section{Late Location-Dependent Modulation of the Extrastriate Areas}

Longer latency modulation of extrastriate processing reflected in target-related difference waves showed a prominent reduction of the activation over extrastriate areas in the 250- to $350-\mathrm{msec}$ latency range. This time range corresponds to the long-latency N2 response, a visual response evoked by attended stimuli and involved in postdiscrimination processes (Luck, 1995; Smid et al., 1999; Wijers et al., 1997). The attenuation of phasic modulation of extrastriate activation at later stages of visual processing therefore supports the involvement of the PFC in the postselection analysis of target features. The impairment in feature selection revealed by electrophysiological measures was paralleled by an attentional impairment in behavioral measures. Indeed, prefrontallesioned patients were slower and less accurate than controls in detecting target stimuli.

The temporal dynamics of PFC attention-specific modulations are in agreement with single-unit recordings in monkeys revealing enhanced prefrontal target-related 
activity $140 \mathrm{msec}$ after stimulus onset (Rainer et al., 1998) and top-down activation of inferior temporal neurons 178-300 msec after target detection (Tomita et al., 1999). The target $\mathrm{P} 3 \mathrm{~b}$ response, generated at about $400 \mathrm{msec}$ post stimulus onset, was spared in the frontal-lesioned patients. This seems to be at odds with the disruption of ipsilesional $\mathrm{P} 3 \mathrm{~b}$ activity observed when patients performed a bifield visual discrimination task (cf. Barceló et al., 2000). One potential explanation is that attending to only one visual hemifield, even if this is the contralesioned hemifield, would make the updating and categorization of targets comparatively more efficient than attending to both hemifields would-hence, the apparently normal $\mathrm{P} 3 \mathrm{~b}$ responses.

The interaction of the PFC and the visual extrastriate areas is well documented by means of single-cell recordings (Rainer et al., 1998; Tomita et al., 1999), lesion studies (Barceló et al., 2000; Knight, 1997; Nielsen-Bohlman \& Knight, 1999), and blood flow data (Büchel \& Friston, 1997; Chawla et al., 1999; Corbetta et al., 1991; de Fockert et al., 2001; Kastner et al., 1999; McIntosh et al., 1994; Rees et al., 1997; Tootell et al., 1998). Furthermore, monkey studies showing direct projections from the PFC to the inferior temporal cortex (Desimone \& Duncan, 1995; Tomita et al., 1999; Webster \& Ungerleider, 1994) provide a potential anatomical pathway to support the interaction between these brain areas. The results of the present study support the crucial role of the PFC in the modulation of the visual extrastriate cortex and suggest two different mechanisms by which this modulation is carried out. At early stages of visual processing, the PFC exerts a predominantly tonic prestimulus modulation over visual areas necessary to hold a working memory template. At later stages of visual processing, the PFC exerts an attention-dependent input to the extrastriate cortex necessary for poststimulus feature selection, as manifested by the SN in controls. Finally, when a designated target event is detected, the PFC exerts a third phasic input signifying successful template matching.

The overall pattern of results supports a key role of the human PFC in visual attention control and is in accord with the large body of work developed by Patricia GoldmanRakic on the critical function played by the PFC in executive control. The present study reveals a complex series of intrahemispheric interactions within a fronto-posterior cortical network, which results in at least three distinct prefrontal-dependent mechanisms of attentional control: (1) an attention-independent excitatory input to the extrastriate cortex (P1), (2) an early attention-dependent excitatory input to the extrastriate cortex (SN), and (3) a late attention-dependent excitatory input to the extrastriate cortex (target N2). The finding of multiple distinct PFC modulations of human visual attention in the first few hundred milliseconds of stimulus processing provides a rich control mechanism for the selection and analysis of environmental inputs, an idea that GoldmanRakic would likely embrace in her view of the importance of the PFC in cognitive control.

\section{REFERENCES}

Baas, J. M., Kenemans, J. L., \& Mangun, G. R. (2002). Selective attention to spatial frequency: An ERP and source localization analysis. Clinical Neurophysiology, 113, 1840-1854.

Barceló, F., SuWAZONO, S., \& KNIGHT, R. T. (2000). Prefrontal modulation of visual processing in humans. Nature Neuroscience, 3, 399-403.

BrefCZYNSKI, J. A., \& DeYoe, E. A. (1999). A physiological correlate of the "spotlight" of visual attention. Nature Neuroscience, 2, 370374.

BüCHEL, C., \& FrISTON, K. J. (1997). Modulation of connectivity in visual pathways by attention: Cortical interactions evaluated with structural equation modeling and fMRI. Cerebral Cortex, 7, 768778 .

Chawla, D., Rees, G., \& Friston, K. J. (1999). The physiological basis of attentional modulation in extrastriate visual areas. Nature Neuroscience, 2, 671-676.

CLARK, V. P., \& Hillyard, S. A. (1996). Spatial selective attention affects early extrastriate but not striate components of the visual evoked potential. Journal of Cognitive Neuroscience, 8, 387-402.

CorbetTA, M. (1998). Frontoparietal cortical networks for directing attention and the eye to visual locations: Identical, independent, or overlapping neural systems? Proceedings of the National Academy of Sciences, 95, 831-838.

Corbetta, M., Kincade, J. M., Ollinger, J. M., McAvoy, M. P., \& Shulman, G. L. (2000). Voluntary orienting is dissociated from target detection in human posterior parietal cortex. Nature Neuroscience, 3, 292-297.

Corbetta, M., Miezin, F. M., Dobmeyer, S., Shulman, G. L., \& PeTERSEN, S. E. (1991). Selective and divided attention during visual discriminations of shape, color, and speed: Functional anatomy by positron emission tomography. Journal of Neuroscience, 11, 2383-2402.

Corbetta, M., Miezin, F. M., Shulman, G. L., \& Petersen, S. E. (1993). A PET study of visuospatial attention. Journal of Neuroscience, 13, 1202-1226.

Daffner, K. R., Scinto, L. F., Weitzman, A. M., Faust, R., Rentz, D. M., Budson, A. E., \& Holcomb, P. J. (2003). Frontal and parietal components of a cerebral network mediating voluntary attention to novel events. Journal of Cognitive Neuroscience, 15, 294-313.

De Fockert, J. W., Rees, G., Frith, C. D., \& LaVIE, N. (2001). The role of working memory in visual selective attention. Science, 291, 18031806.

Desimone R., \& DunCan, J. (1995). Neural mechanisms of selective visual attention. Annual Review of Neuroscience, 18, 193-222.

Friedman-Hill, S. R., Robertson, L. C., \& Treisman, A. (1995). Parietal contributions to visual feature binding: Evidence from a patient with bilateral lesions. Science, 269, 853-855.

Gitelman, D. R., Nobre, A. C., PARRISH, T. B., LABAR, K. S., Kim, Y.-H., Meyer, J. R., \& Mesulam, M.-M. (1999). A large-scale distributed network for covert spatial attention: Further anatomical delineation based on stringent behavioural and cognitive controls. Brain, $\mathbf{1 2 2}$, 1093-1106.

HARTER, M. R., \& Aine, C. J. (1984). Brain mechanisms of visual selective attention. In R. Parasuraman \& D. R. Davies (Eds.), Varieties of attention (pp. 293-321). Orlando, FL: Academic Press.

Haxby, J. V., Horwitz, B., Ungerleider, L. G., Maisog, J. M., Pietrini, P., \& Grady, C. L. (1994). The functional organization of human extrastriate cortex: A PET-rCBF study of selective attention to faces and locations. Journal of Neuroscience, 14, 6336-6353.

Heinze, H. J., Mangun, G. R., Burchert, W., Hinrichs, H., SCHOlZ, M., Münte, T. F., Gös, A., Scherg, M., Johannes, S., Hundeshagen, H., Gazzaniga, M. S., \& Hillyard, S. A. (1994). Combined spatial and temporal imaging of brain activity during visual selective attention in humans. Nature, 372, 543-546.

Hillyard, S. A., \& ANLlo-Vento, L. (1998). Event-related brain potentials in the study of visual selective attention. Proceedings of the National Academy of Sciences, 95, 781-787.

Hillyard, S. A., Mangun, G. R., WoldDORF, M. G., \& LuCK, S. J. (1995). Neural systems mediating selective attention. In M. S. Gazzaniga (Ed.), The cognitive neurosciences (pp. 665-681). Cambridge, MA: MIT Press. 
Hillyard, S. A., \& Münte, T. F. (1984). Selective attention to color and location: An analysis with event-related brain potentials. Perception \& Psychophysics, 36, 185-198.

Hopfinger, J. B., BuONocoRE, M. H., \& Mangun, G. R. (2000). The neural mechanisms of top-down attentional control. Nature Neuroscience, 3, 284-291.

Kastner, S., Pinsk, M. A., De Weerd, P., Desimone, R., \& UngerLEIDER, L. G. (1999). Increased activity in human visual cortex during directed attention in the absence of visual stimulation. Neuron, 22, 751-761.

KNIGHT, R. T. (1984). Decreased response to novel stimuli after prefrontal lesions in man. Electroencephalography \& Clinical Neurophysiology, 59, 9-20.

KNIGHT, R. T. (1997). Distributed cortical network for visual attention. Journal of Cognitive Neuroscience, 9, 75-91.

LUCK, S. J. (1995). Multiple mechanisms of visual-spatial attention: Recent evidence from human electrophysiology. Behavioural Brain Research, 71, 113-123.

MANGUN, G. R. (1995). Neural mechanisms of visual selective attention. Psychophysiology, 32, 4-18.

MANGUN, G. R., BUONOCORE, M. H., GiRelli, M., \& JHA, A. P. (1998). ERP and fMRI measures of visual spatial selective attention. Human Brain Mapping, 6, 383-389.

MANGUN, G. R., \& Hillyard, S. A. (1993). Modulations of sensoryevoked brain potentials indicate changes in perceptual processing during visual-spatial priming. Journal of Experimental Psychology: Human Perception \& Performance, 17, 1057-1074.

Martinez, A., Anllo-Vento, L., Sereno, M. I., Frank, L. R., BuXTon, R. B., Dubowitz, D. J., Wong, E. C., Hinrichs, H., Heinze, H. J., \& HILLYARD, S. A. (1999). Involvement of striate and extrastriate visual cortical areas in spatial attention. Nature Neuroscience, 2, 364-369.

McIntosh, A. R., Grady, C. L., UnGerleider, L. G., HAXBY, J. V., RAPOPORT, S. I., \& Horwitz, B. (1994). Network analysis of cortical visual pathways mapped with PET. Journal of Neuroscience, $\mathbf{1 4}$ 655-666.

MESUlam, M.-M. (1999). Spatial attention and neglect: Parietal, frontal and cingulate contributions to the mental representation and attentional targeting of salient extrapersonal events. Philosophical Transactions of the Royal Society of London: Series B, 354, 1325-1346.

MiLleR, E. K. (1999). The prefrontal cortex: Complex neural properties for complex behavior. Neuron, 22, 15-17.

Nielsen-Bohlman, L. C., \& KNIGHT, R. T. (1999). Prefrontal cortical involvement in visual working memory. $\underline{\text { Cognitive Brain Research, } \mathbf{8}}$ 299-310.

Posner, M. I., \& Dehaene, S. (1994). Attentional networks. Trends in Cognitive Neurosciences, 17, 75-79.

Rainer, G., AsaAd, W. F., \& Miller, E. K. (1998). Selective representation of relevant information by neurons in the primate prefrontal cortex. Nature, 393, 577-579.
Rees, G., Frackowiak, R., \& Frith, C. (1997). Two modulatory effects of attention that mediate object categorization in human cortex. Science, 275, 835-838.

REYNOLDS, J. H., PASTERNAK, T., \& Desimone, R. (2000). Attention increases sensitivity of V4 neurons. Neuron, 26, 703-714.

Sereno, M. I., Dale, A. M., Reppas, J. B., KWONG, K. K., Belliveau, J. W., Brady, T. J., Rosen, B. R., \& Tootell, R. B. (1995). Borders of multiple visual areas in humans revealed by functional magnetic resonance imaging. Science, 268, 889-893.

SMID, H. G. O. M., JАKOB, A., \& HEINZE, H.-J. (1999). An event-related brain potential study of visual selective attention to conjunctions of color and shape. Psychophysiology, 36, 264-279.

Suwazono, S., MaCHADO, L., \& KNIGHT, R. T. (2000). Predictive value of novel stimuli modifies visual event-related potentials and behavior. Clinical Neurophysiology, 111, 29-39.

Tomita, H., Ohbayashi, M., Na KaHARa, K., Hasegawa, I., \& MiYaSHITA, Y. (1999). Top-down signal from prefrontal cortex in executive control of memory retrieval. Nature, 401, 699-703.

Tootell, R. B., Hadjikhani, N., Hall, E. K., Marrett, S., VanduFfel, W., Vaughan, J. T., \& Dale, A. M. (1998). The retinotopy of visual spatial attention. Neuron, 21, 1409-1422.

Treue, S. (2003). Visual attention: The where, what, how and why of saliency. Current Opinion in Neurobiology, 13, 428-432.

Valdes-Sosa, M., Bobes, M. A., Rodríguez, V., \& Pinilla, T. (1998). Switching attention without shifting the spotlight: Object-based attentional modulation of brain potentials. Journal of Cognitive Neuroscience, 10, 137-151.

Vandenberghe, R., Duncan, J., Dupont, P., Ward, R., Poline, J.-B., Bormans, G., Michiels, J., Mortelmans, L., \& Orban, G. A. (1997). Attention to one or two features in left or right visual field: A positron emission tomography study. Journal of Neuroscience, 17, 3739-3750.

WeBster, M. J., \& UNGERLEIDER, L. G. (1994). Neuroanatomy of visual attention. In R. Parasuraman (Ed.), The attentive brain (pp. 19-34). Cambridge, MA: MIT Press.

WiJers, A. A., Lange, J. J., Mulder, G., \& Mulder, L. J. (1997). An ERP study of visual spatial attention and letter target detection for isoluminant and nonisoluminant stimuli. Psychophysiology, 34, 553-565.

Wijers, A. A., Mulder, G., Okita, T., Mulder, L. J., \& Scheffers, M. K. (1989). Attention to color: An analysis of selection, controlled search, and motor activation, using event-related potentials. Psychophysiology, 26, 89-109.

WoldorfF, M. G., Fox, P. T., Matzke, M., Lancaster, J. L., Veeraswamy, S., Zamarripa, F., Seabolt, M., Glass, T., Gao, J. H., MarTIN, C. C., \& JERABEK, P. (1997). Retinotopic organization of early visual spatial attention effects as revealed by PET and ERPs. Human Brain Mapping, 5, 280-286.

(Manuscript received August 15, 2004; revision accepted for publication December 20, 2004.) 